\title{
Mathematical relationship between glass transition temperature and water activity of cellular and non-cellular food systems
}

\author{
Nguyen, T. K. ${ }^{\text {a,c }}$; Khalloufi, S. ${ }^{\text {b,* }}$; Ratti, C. ${ }^{\text {a,b }}$ \\ a Institute of Nutrition and Functional Foods (INAF), Université Laval, QC, Canada, G1V 0A6 \\ b Soils and Agri-Food Engineering Department, Université Laval, QC, Canada, G1V 0A6 \\ c Food Science Department, Université Laval, QC, Canada, G1V 0A6 \\ *E-mail of the corresponding author: seddik.khalloufi@ulaval.ca
}

\begin{abstract}
Cellular and non-cellular-solid food systems were used to obtain experimental data of $a_{w}$ and $\mathrm{Tg}$ as a function of moisture content during drying. GAB, Gordon-Taylor, and Khalloufi-Ratti models were used to obtain the state diagrams of the four food systems investigated. The results suggest that the $G A B$ and Khalloufi-Ratti models can successfully be used to capture the experimental data. In terms of plasticizing effect, it seems that cellular and non-cellular systems have comparable values. Although the number of food samples explored in this study was limited, it is suggested that the chemical composition could have more impact on $\mathrm{Tg}$ and stability than the presence of cell structures.
\end{abstract}

Keywords: Isotherms; Glass Transition; Cellular and Non-Cellular Food Systems; Modeling. 


\section{Introduction}

Several mathematical models are available for describing sorption isotherms. The widely used model for food matrices is Guggenheim-Anderson-de Boer (GAB) [1]. This model is based on the monolayer moisture concept, and may be used to provide estimative values of critical moisture content and relative humidity for safe storage of dried foods [2]. With repect to bio-material, the Flory-Huggins model has been also able to properly describe sorption isotherms $[3,4]$.

The concept of glass transition has been widely applied to food, polymer, material and pharmaceutical sciences to relate physical, chemical and structural changes to the physical state of the material [2]. In general, the $T g$ value depends on the thermal history of the material, the molecular weight of the polymer chains, the presence of a plasticizer, the degree of crystallinity and sample composition [5]. Water, with low molecular weight and low $\mathrm{Tg}$ ($135^{\circ} \mathrm{C}$ ) [6], is the most important plasticizing agent in foodstuffs, increasing the flexibility of matrix and decreasing $\mathrm{Tg}$. Water plasticization effect may be well represented in foods by the Gordon-Taylor (G-T) equation [7] and, for polymer blends, by the Couchman and Karasz (C-K) equation [8]. Khalloufi et al. (2000) developed an equation to represent glass transition temperature of fruit powders as a function of water activity, which proposes a sigmoid rather than linear representation of $\mathrm{Tg}$ in the whole range of water activity [9].

Both water activity and glass transition have been used extensively in the literature to evaluate storage stability $[10,11]$. The combination of these two parameters can be used to obtain the state diagram. [12] which can help to select packaging material and optimizing ingredient in food formulation. Furthermore, the state diagram can be used to design and optimize drying equipment, as well as to model and control processing, i.e. determination of the end-point of drying or assessing the energy requirement by choosing optimum temperature and moisture content to avoid undesirable effects [13]. So far, the state diagrams for various foodstuffs have been reported in literature [2, 14, 15, 16]. However, none of this data compared cellular to non cellular systems. In addition, state diagams for maltodextrins, carrots and potatoes are rare in the open littertaure [17].

The objective of the current study was to develop the state diagrams for potato, carrot and two agar-maltodextrin model systems (AM DE19 and AM DE36), by measuring the glass transition temperature and water sorption isotherms. The GAB and Flory-Huggins equations were used to describe the sorption isotherms, while the G-T equation, for the glass transition temperature. The relationship between $T g$ and $a_{w}$ was established from Khalloufi-Ratti model [9]. State diagrams for the four products were built from mathematical representations for $\mathrm{Tg}, a_{w}$ and moisture content and compared with experimental data. 


\section{Materials and Methods}

\subsection{Material and processing}

Maltodextrin was provided by Laboratory Mat (Quebec city, Canada). Agar was delivered by Becton Dickinson and Company (Sparks, MD 21152 USA). White potatoes (W1386 variety) were provided by Gosselin G2 Inc. (Quebec City, Canada). Carrots were purchased directly from a local market in Quebec, Canada (ATV farms, Quebec). Two cellular systems (potato and carrot) and two non-cellular systems (water-agar-maltodextrin: AM DE19 and AM DE36) were used in this study. The non-cellular models were prepared in a mass proportion of water/agar/maltodextrin of 1/0.015/0.15. Cylindrical samples were made and cut from random positions within the sample with a hollow punch in $1 \mathrm{~cm}$-diameter by $4 \mathrm{~cm}$ long cylinders. Samples were dried by convection in an Armfield tunnel dryer (Model UOP8G, Hampshire, UK) with air at $55^{\circ} \mathrm{C}$ and air speed of $1.6 \mathrm{~m} / \mathrm{s}$, until constant weight.

\subsection{Sorption Isotherms}

Materials with various moisture contents and water activities were obtained by rehumidification of powdered air-dried samples at various relative humidity levels over saturated salt solutions in desiccators at $25^{\circ} \mathrm{C}$. Relative humidity of the saturated solutions was checked by measuring their water activity with an Aqualab (model series 3, Decagon Devices Inc, USA).

\subsection{Glass transition temperature $(\mathrm{Tg})$}

The glass transition temperature of equilibrated samples was measured with a Differential Scanning Calorimeter DSC Pyris 1 (Perkin Elmer, Shelton, CT, USA).

\subsection{Mathematical modeling}

The mathematical relationships used in this contribution are presented in Table 1.

Table 1. Mathematical relationships between moisture content $(X)$, water activity $\left(a_{w}\right)$, and/or glass transition temperature ( $\mathrm{Tg})$

\begin{tabular}{lcc}
\hline \multicolumn{1}{c}{ Model \& Refernce } & Mathematical expression \\
\hline GAB [1] & $X=\frac{\left(X_{m} Y K a_{w}\right)}{\left(1-K a_{w}\right)\left(1-K a_{w}+Y K a_{w}\right)}$ \\
Flory-Huggins [3] & $\ln a_{w}=\ln \left(1-\phi_{p}\right)+\phi_{p}-\frac{\phi_{p}}{N}+\chi \phi_{p}^{2}$ \\
Gordon-Taylor [7] & $T g=\frac{(1-w) T g_{s}+k w T g_{w}}{(1-w)+k w}$ \\
Khalloufi-Ratti [9] & $T g=\frac{A a_{w}^{2}+B a_{w}+C}{D a_{w}^{2}+E a_{w}+1}$ \\
\hline
\end{tabular}


Mathematical relationship between glass transition temperature and water activity of cellular and non-cellular food systems

\section{Results and discussion}

Figure 1 shows the results obtained by using the Flory-Huggins model to describe water activity as a function of volume fraction. The Flory-Huggins model was satisfactory for carrot, but not for other food systems investegated in this contribution (e.g. AM DE19, AM DE36 and potato). According to Kocherbitov (2016), the Flory-Huggins equation is not able to describe the glassy part of water sorption isotherms of some polymers [4]. As the FloryHuggins model was not suitable for all the four food systems, the GAB model model will be used for the next steps of this study (Figure 2 and Table2).

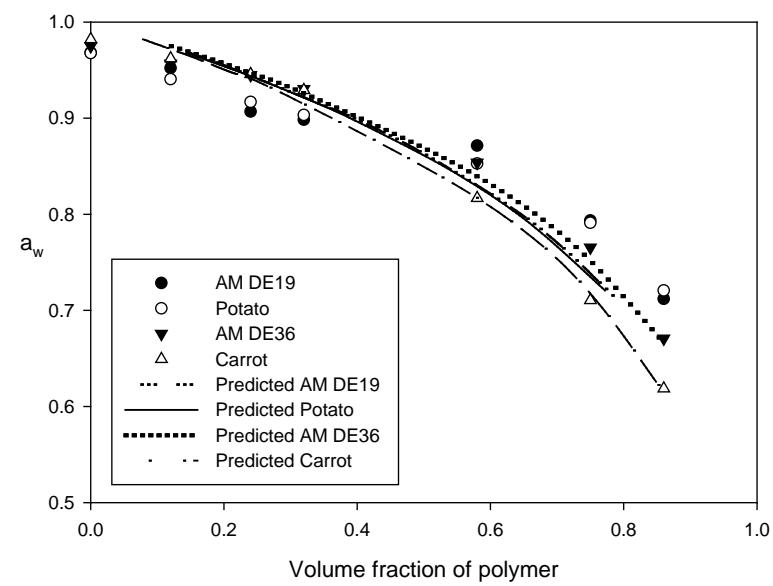

Figure 1. Comparison between experimental data and predictions of Flory-Huggins model

The $T g$ values for carrots and potatoes, obtained in this investigation, are close to those reported by Karmas et al. (1992). Georget et al. (1999) detected two glass transition temperatures for carrot material associated with two phases: a sugar-rich phase and a cell wall-rich phase. The $\mathrm{Tg}$ value found in the current study is considered to be in agreement with those of the sugar-rich phase but lower than those of the cell wall rich phase [17]. Three main saccharides occurring commonly in carrot roots are glucose, fructose and sucrose [18], thus the glass transition temperature in carrots are related to $\mathrm{Tg}$ for those three types of sugars. The $\mathrm{Tg}$ value of carrots is also similar to the one of rich glucose-fructose fruit such as raspberry $\left(\mathrm{Tg}_{s}=42.62^{\circ} \mathrm{C}\right)$ [2]. In the case of potato, the $\mathrm{Tg}$ value in both native or non-native potato starch reported in literature $\left(120^{\circ} \mathrm{C}\right)$ is much higher than the experimental data found in the present study $\left(49.8 \pm 12.3^{\circ} \mathrm{C}\right)$ for dry potato $[5,19]$. Significant differences between $\mathrm{Tg}$ 
values of dried potato tuber and potato starch was also observed by Chirife et al. (1996). According to Biliaderis et al. (1986), the absence of a clearly detectible glass transition in native starch is due to the amorphous chains locked by crystalline domains, the decrease of mobility of the amorphous chain by physical crosslinks and the presence of inter-crystalline phases that do not follow normal thermal behavior. The constants $T g_{s}$ and $k$ of the GordonTaylor model (Eq. 3) were estimated by non-linear regression for the four types of sample materials, which results are shown in Table 2. The $k$ values of the four products studied in the present work were in the range reported for fruits and vegetables such as onion $(k=4.3)$, raspberry $(\mathrm{k}=4.7)$, strawberry $(k=4.3)$, mango $(k=4.5)$, kiwifruit $(k=4.9)$, gooseberry $(k$ $=5.7)$ and banana $(k=6.1)[2,14,15,16,20,21]$. The $k$ value is an estimate of the plasticization effect of water, which means the strength of interaction between water and the food solids. Higher values indicate a greater plasticizing effect of water on solids [12]. From Table 2, it can be observed that water has a greater plasticizing effect on potato than on carrot. For carrot, this plasticizing effect of water was the lowest among the four products.

Table 2. Parameters involved in the Gordon-Taylor model (Eq. 3, Table 1)

\begin{tabular}{cccc}
\hline & $\operatorname{Tg}\left({ }^{\circ} \mathrm{C}\right)$ & $k$ & $\mathrm{r}^{2}$ \\
\hline AM DE19 & 108.4 & 4.9 & 0.97 \\
AM DE36 & 81.9 & 5.2 & 0,99 \\
Potato & 76.2 & 6.8 & 0.99 \\
Carrot & 35.2 & 4.3 & 0.96 \\
\hline
\end{tabular}

State diagrams (Figure 2) was built by using experimental data and the predictions obtained from the mathematical model of GAB expression (for water sorption) and from KhalloufiRatti model (for glass transition temperature). As it can be seen, the predictions obtained by both models are in agreement with the experimental data. The monolayer water content $\left(X_{m}\right)$ obtained from the $G A B$ equation is an important parameter for stability of foods, which depends on chemical composition and temperature of the material. A product is most stable below $X_{m}$ where rates of deteriorative reactions are minimal [22]. At temperature of $25^{\circ} C$, the estimated values of the parameter $X_{m}$ is $0.049 \mathrm{~kg} / \mathrm{kg}(\mathrm{db})$ for $A M D E 19,0.065 \mathrm{~kg} / \mathrm{kg}(\mathrm{db})$ for $A M D E 36,0.056 \mathrm{~kg} / \mathrm{kg}(\mathrm{db})$ for potato. These values were found to be lower than the one 
for carrot, which was $0.195 \mathrm{~kg} / \mathrm{kg}(\mathrm{db})$. These results are close to those reported by Kiranoudis et al. (1993) which found that the $X_{m}$ value of potato was 0.087 and that of carrot was $0.21 \mathrm{~kg} / \mathrm{kg}(\mathrm{db})$.

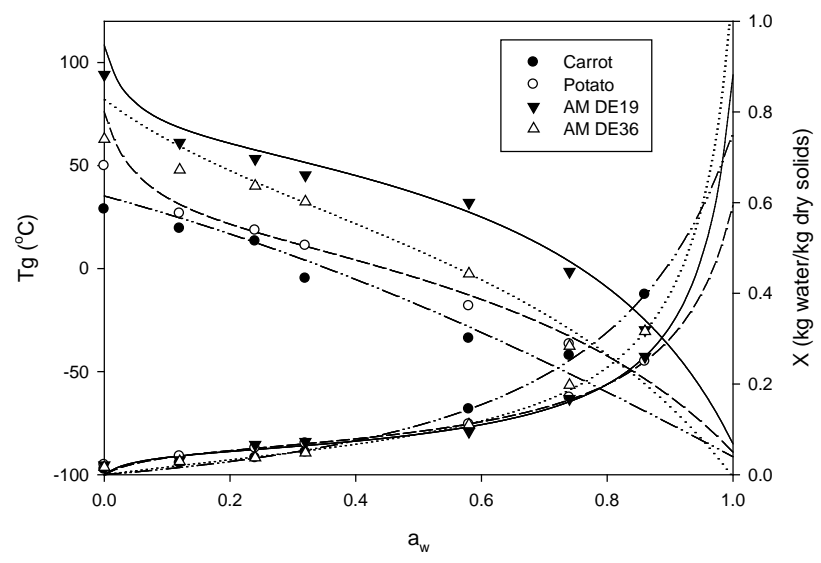

Figure 2. State diagram of cellular and non-cellular food materials. Lines are predicted data obtained by using GAB and Khalloufi-Ratti models

The relationship between $a_{w}$ and $T g$ has been reported to be sigmoid [11]. The KhalloufiRatti model [9] allows capturing this sigmoidal profile for all the food sytems investigated in this study (Figure 2). This model is a combination of paramters obtained previously from Gordon-Taylor and GAB models (Table 1).

Table 2 shows the critical water content and critical water activity of four products at $4^{\circ} \mathrm{C}$ and $25^{\circ} \mathrm{C}$ obtained from state diagram (Figure 2). The critical water content (CWC) and critical water activity (CWA) are the value at which the glass transition occurs, at a determined storage temperature of the product. These value are helpful to show the dependence of $T g$ of product on moisture content or water activity. For example, if the AM DE19 is conserved at $25^{\circ} \mathrm{C}$, the CWC and CWA at which the glassy system AMDE19 transformed to a rubbery state is $0.096 \mathrm{~kg} / \mathrm{kg} \mathrm{dm}$ and 0.536 , respectively. According to the water activity concept, foods are most stable at their monolayer moisture content. While the glass transition concept propose that amorphous matrix are more stable at or below the corresponding $\operatorname{Tg}[10,13]$. 
Nguyen, T. K.; Khalloufi, S.; Ratti, C.

Table 3. Critical water activity (awc) and Critical water content $\left(X_{c}\right)$

\begin{tabular}{lcccc} 
& \multicolumn{2}{c}{$4^{\circ} \mathrm{C}$} & \multicolumn{2}{c}{$25^{\circ} \mathrm{C}$} \\
\cline { 2 - 5 } & $a_{w c}$ & $X_{c}$ & $a_{w c}$ & $X_{c}$ \\
\hline AM DE19 & 0.6765 & 0.1336 & 0.5389 & 0.0966 \\
AM DE36 & 0.4883 & 0.0865 & 0.3439 & 0,0567 \\
Potato & 0.3416 & 0.0711 & 0.1453 & 0.0450 \\
Carrot & 0.3303 & 0.0493 & 0.1105 & 0.0145 \\
\hline
\end{tabular}

\section{Conclusions}

Water activity $\left(a_{w}\right)$ and glass transition temperature $(\mathrm{Tg})$ are relevant concepts to predict the stability of food products. The Flory-Huggins model was able to describe the sorption isotherm of carrot but not for other food systems investigated in this study. GAB and Khalloufi-Ratti models were succusfuly able to capture the experimental profile of cellular and non cellular food systems. These two mathemetaical models can be used to construct the state diagrams which describes the physical states as a function of water activity and water content of foods. This state diagram can be used as a significant tool to help food scientists, food engineers and food-packaging researchers to develop processing protocols and selecting storage conditions. In terms of plasticizing effect of water, it seems that cellular and noncellular systems have comparable values. Although the number of food samples used in this study was limited, this contribution suggests that the chemical composition of food systems could have more impact on their $\mathrm{Tg}$ and stability than the presence of cell structures. Further investigations with diverse food systems are required to confirm this preliminary finding.

\section{References}

[1] Al-Muhtaseb, A.H.; McMinn, W.A.M.; Magee, T.R.A. Moisture Sorption Isotherm Characteristics of Food Products: A Review. Food and Bioproducts Processing. 2002, 80, 118-128.1

[2] Syamaladevi, R.M.; Sablani, S.S.; Tang, J.; Powers, J.; Swanson, B.G. State diagram and water adsorption isotherm of raspberry (Rubus idaeus). Journal of Food Engineering. 2009, 91, 460-467.2

[3] Hancock, B.; Zografi, G. The Use of Solution Theories for Predicting Water Vapor Absorption by Amorphous Pharmaceutical Solids: A Test of the Flory-Huggins and Vrentas Models. Pharmaceutical Research. 1993, 10, 1262-1267.3

[4] Kocherbitov, V. The nature of nonfreezing water in carbohydrate polymers. Carbohydr Polym. 2016, 150, 353-8.4

[5] Thiewes, H.J.; Steeneken, P.A.M. The glass transition and the sub- T g endotherm of amorphous and native potato starch at low moisture content. Carbohydrate Polymers. 1997, 32, 123-130.30

[6] Johari, G.P.; Andreas, H.; Erwin, M. The glass-liquid transition of hyperquenched 
Mathematical relationship between glass transition temperature and water activity of cellular and non-cellular food systems

water. Nature. 1987, 330, 552.31

[7] Gordon, M.; Taylor, J.S. Ideal copolymers and the second-order transitions of synthetic rubbers. i. non-crystalline copolymers. Journal of Applied Chemistry. 1952, 2, 493500.32

[8] Couchman, P.R.; Karasz, F.E. A Classical Thermodynamic Discussion of the Effect of Composition on Glass-Transition Temperatures. Macromolecules. 1978, 11, 117-119.33

[9] Khalloufi, S.; El-Maslouhi, Y.; Ratti, C. Mathematical Model for Prediction of Glass Transition Temperature of Fruit Powders. Journal of Food Science. 2000, 65, 842-848.34

[10] Chirife, J.; del Pilar Buera, M.; Labuza, T.P. Water activity, water glass dynamics, and the control of microbiological growth in foods. Critical Reviews in Food Science and Nutrition. 1996, 36, 465-513.3

[11] Roos, Y.; Karel, M. Applying state diagrams to food processing and development. Food technology. 1991, 45, 66, 68.35

[12] Sablani, S.S.; Syamaladevi, R.M.; Swanson, B.G. A Review of Methods, Data and Applications of State Diagrams of Food Systems. Food Engineering Reviews. 2010, 2, 168-203.29

[13] Sablani, S.S.; Kasapis, S.; Rahman, M.S. Evaluating water activity and glass transition concepts for food stability. Journal of Food Engineering. 2007, 78, 266-271.38

[14] Moraga, G.; Martínez-Navarrete, N.; Chiralt, A. Water sorption isotherms and phase transitions in kiwifruit. Journal of Food Engineering. 2006, 72, 147-156.40

[15] Sá, M.M.; Sereno, A.M. Glass transitions and state diagrams for typical natural fruits and vegetables. Thermochimica Acta. 1994, 246, 285-297.42

[16] Zhao, J.-H.; Liu, F.; Wen, X.; Xiao, H.-W.; Ni, Y.-Y. State diagram for freeze-dried mango: Freezing curve, glass transition line and maximal-freeze-concentration condition. Journal of Food Engineering. 2015, 157, 49-56.43

[17] Georget, D.M.R.; Smith, A.C.; Waldron, K.W. Thermal transitions in freeze-dried carrot and its cell wall components. Thermochimica Acta. 1999, 332, 203-210.47

[18] Baranski, R.; Allender, C.; Klimek-Chodacka, M. Towards better tasting and more nutritious carrots: Carotenoid and sugar content variation in carrot genetic resources. Food Research International. 2012, 47, 182-187.55

[19] Farahnaky, A.; Farhat, I.A.; Mitchell, J.R.; Hill, S.E. The effect of sodium chloride on the glass transition of potato and cassava starches at low moisture contents. Food Hydrocolloids. 2009, 23, 1483-1487.56

[20] Wang, H.; Zhang, S.; Chen, G. Glass transition and state diagram for fresh and freezedried Chinese gooseberry. Journal of Food Engineering. 2008, 84, 307-312.

[21] Moraga, G.; Talens, P.; Moraga, M.J.; Martínez-Navarrete, N. Implication of water activity and glass transition on the mechanical and optical properties of freeze-dried apple and banana slices. Journal of Food Engineering. 2011, 106, 212-219.

[22] Rahman, M.S. State diagram of foods: Its potential use in food processing and product stability. Trends in Food Science \& Technology. 2006, 17, 129-141.12 